\title{
Influência da escuta contextualizada na percepção da intensidade do desvio vocal
}

\section{Influence of clinical context in characterization of severity of vocal deviation}

\author{
Flávia Pereira da Costa ${ }^{1}$, Rosiane Yamasaki', Mara Behlau ${ }^{1}$
}

\begin{abstract}
RESUMO
Objetivo: Verificar se a escuta clínica contextualizada influencia a avaliação perceptivo-auditiva da intensidade do desvio vocal. Métodos: Foram selecionados 22 registros vocais de 12 mulheres e dez homens, faixa etária de 25 a 75 anos, pré e pós fonoterapia. A amostra vocal foi analisada por duas fonoaudiólogas especialistas em voz. A avaliadora 1 era a terapeuta dos pacientes e realizou a escuta clínica contextualizada e a avaliadora 2 não conhecia nenhum dos pacientes e realizou a escuta do sinal sonoro. O material de fala foi a emissão da vogal sustentada "é" e a contagem de números de um a dez. A estratégia utilizada foi a de marcar o grau geral de desvio vocal em uma escala analógica visual de 100 mm. Resultados: Na vogal sustentada, no momento pré-fonoterapia, a avaliadora 1 produziu a média de 53,8 pontos (faixa de 17 a 100), enquanto a avaliadora 2 produziu a média de 62,8 (faixa de 32 a 100). No momento pós fonoterapia, a média da avaliadora 1 foi de 22,8 pontos (faixa de 7 a 47) e a da avaliadora 2 foi de 51,9 pontos (faixa de 28 a 92), para vogal sustentada. Para a fala encadeada, o momento pós fonoterapia foi o único que se encontrou diferença, sendo que a avaliadora 1 teve média de 18,41 pontos (faixa de 5 a 55 ) e a avaliadora 2 , média de 43,55 pontos (faixa de 18 a 80 ). Conclusão: A vogal sustentada sofreu mais influência do conhecimento de dados demográficos e de diagnóstico vocal do que a fala encadeada.
\end{abstract}

Descritores: Voz; Qualidade da voz; Distúrbios da voz; Fonoterapia; Dados demográficos

\begin{abstract}
Purpose: We verify if the clinical context interferes in the assessment of vocal deviation, considering the overall degree of severity. Methods: We selected 22 voice recordings of 12 women and 10 men diagnosed with organic or functional dysphonia, aged between 25 and 75 years old, pre-and post-therapy. The vocal sample was analyzed by two SLP voice specialists, one of which (SLP-1) was the patients' therapist and conducted a contextualized clinical assessment. On the other hand, the second voice specialist (SLP-2), did not know any of the patients and conducted the assessment only by listening to the recordings. The speech material used was the sustained vowel / $/$ / and continuous speech (number counting 1 to 10). The overall degree of vocal deviation should be marked on a visual analog scale of $100 \mathrm{~mm}$. Results: In the sustained vowel the SLP-1 produced an average of 53.8 on pre-therapy evaluation (range 17 to 100), while the SLP-2 produced an average of 62.8 (range 32 to 100 ). In the post-therapy assessments, the average was 22.8 for SLP-1 (range 7 to 47), and 51.9 for SLP-2 (range 28 to 92). To the continuous speech the post-therapy assessments was the only with significant difference, the SLP-1 produced an average of 18.41 (range 5 to 55) while the SLP-2 produced an average of 43.55 (range 18 to 80 ). Conclusion: The sustained vowel suffers more influences of demographic data and diagnostic vocal than continuous speech.
\end{abstract}

Keywords: Voice; Voice quality; Voice disorders; Speech therapy; Demographic data

Trabalho realizado no Centro de Estudos da Voz - CEV - São Paulo (SP), Brasil, como pré-requisito para conclusão do Curso de Especialização em Voz. (1) Centro de Estudos da Voz - CEV, São Paulo (SP), Brasil.

Conflito de interesses: Não

Contribuição dos autores: $F P C$ : desenho do estudo, análise dos dados, redação do artigo; $R Y$ : desenho do estudo, análise dos dados, redação e revisão do artigo; $M B$ : orientadora, desenho do estudo, análise dos dados, redação e revisão do artigo.

Endereço para correspondência: Flávia Pereira da Costa. R. Machado Bittencourt, 361/1006, Vila Clementino, São Paulo (SP), Brasil, CEP: 04044-905. E-mail: flaviapdacosta@yahoo.com.br

Recebido em: 09/08/2013; Aceito em: 11/11/2013 


\section{INTRODUÇÃO}

A análise perceptivo-auditiva é a principal forma de avaliação vocal na clínica fonoaudiológica e soberana em relação aos demais métodos de avaliação. Permite a caracterização da qualidade vocal e a quantificação da intensidade do desvio percebido $^{(1)}$. Por meio dessas informações, pode-se realizar inferências sobre a saúde vocal e sobre as características anatomofuncionais da laringe e do trato vocal do falante, o que contribui para o direcionamento do raciocínio clínico do fonoaudiólogo. É uma análise de execução rápida, econômica, não invasiva e robusta ${ }^{(2-4)}$.

A qualidade vocal de indivíduos disfônicos envolve características multidimensionais que podem ser, se não plenamente, parcialmente identificadas pela análise perceptivo-auditiva. Embora seja considerada padrão ouro por muitos pesquisadores, a análise perceptivo-auditiva tem sido alvo de muitas críticas, por ser uma avaliação de natureza subjetiva. Entretanto, estudos mostram que a grande variabilidade nos julgamentos perceptivos está mais relacionada a procedimentos experimentais inadequados ${ }^{(5)}$. Para tornar essa análise mais confiável e robusta, faz-se necessário o controle de importantes fatores que podem interferir em seu resultado, como a experiência e o treinamento do avaliador, o grau de desvio vocal, o tipo de estímulo, a resolução da escala de avaliação, os parâmetros vocais selecionados e a instrução da tarefa de escuta. O controle cuidadoso desses fatores aumenta a confiabilidade da análise e reduz o grau de subjetividade envolvido ${ }^{(1,5,6-12)}$.

Atualmente, tanto em ambientes clínicos como científicos, a modalidade de resposta de escala perceptivo-auditiva mais utilizada é a do formato analógico- visual. Embora a escala numérica seja aparentemente mais fácil de ser utilizada, acaba por concentrar resultados diversos em um mesmo grau de avaliação $^{(5,13)}$ e a marcação na escala analógica visual é mais apropriada para distinguir mudanças discretas na qualidade vocal. Ela é utilizada em escalas de autoavaliação e no CAPE-V (Consensus Auditory- Perceptual Evaluation of Voice - ASHA 2003), protocolo desenvolvido em consenso por especialistas vocais, considerando a tendência moderna na mensuração da percepção humana $^{(14)}$.

A correlação da escala analógica visual com a escala numérica permitiu a obtenção de valores de corte, o que possibilitou a separação de vozes normais de vozes desviadas. No estudo realizado na Finlândia ${ }^{(15)}$, o valor encontrado foi de $35 \mathrm{~mm}$, enquanto que no Brasil ${ }^{(16)}$, o valor obtido foi de $35,5 \mathrm{~mm}$. A semelhança dos valores encontrados nos dois estudos, realizados em países diferentes, mostra que essa forma de avaliação é robusta e confiável.

Além dos fatores de interferência já descritos, a análise auditiva também pode ser influenciada pelo contexto em que a avaliação é realizada. O conhecimento de informações clínicas do paciente, por parte do avaliador, como dados demográficos, gênero, idade, diagnóstico médico ${ }^{(17)}$, histórico da disfonia e evolução clínica, assim como a situação de escuta, que pode ser clínica ou científica ${ }^{(18)}$, podem interferir no resultado da análise auditiva. A avaliação vocal clínica, geralmente é feita na presença do paciente, com o conhecimento de seus dados, do diagnóstico, da condição de saúde vocal e da evolução clínica. Já a escuta cega, geralmente é feita a partir do sinal sonoro, sem acesso a dados básicos do paciente. Como as duas condições de escuta, contextualizada e cega, são muito utilizadas na prática fonoaudiológica, seja para fins clínicos ou científicos, faz-se necessário saber se tais condições influenciam o resultado da análise perceptivo-auditiva.

O objetivo desta pesquisa foi verificar se a escuta clínica contextualizada, caracterizada pelo conhecimento do quadro clínico dos pacientes pelo avaliador, influencia a avaliação perceptivo-auditiva da intensidade do desvio vocal percebido.

\section{MÉTODOS}

O estudo foi realizado no Centro de Estudos da Voz (CEV), com aprovação do Comitê de Ética em Pesquisa do Instituto de Ciências Biomédicas da Universidade de São Paulo (ICBUSP), parecer número 1026.

\section{Amostra vocal}

Foram selecionados, do arquivo de vozes do CEV, 22 registros vocais de pacientes disfônicos, 12 mulheres e dez homens com diagnóstico de disfonia orgânica ou funcional, faixa etária de 25 a 75 anos, com baixa ou alta demanda vocal. A amostra vocal foi retirada do banco de vozes da fonoaudióloga que realizou o tratamento dos pacientes (avaliadora 1) e consistiu da emissão sustentada da vogal "é" e amostra de fala encadeada (contagem de números de um a dez), pré e pós fonoterapia.

A amostra vocal, composta pelos momentos pré e pós fonoterapia, foi apresentada de forma aleatória às duas avaliadoras, com $10 \%$ de repetição, para teste da confiabilidade interna. Dessa forma, cada avaliadora realizou a análise perceptivo-auditiva de 48 emissões sustentadas - vogal "é" - e de 48 emissões de fala encadeada - contagem de um a dez. A análise das emissões sustentadas e da fala encadeada foi realizada em duas sessões de escuta diferentes, com intervalo de tempo de sete dias. A avaliadora 1, evidentemente, conhecia os pacientes e seu curso clínico de tratamento.

\section{Avaliadoras}

A análise perceptivo-auditiva foi realizada por duas avaliadoras, ambas especialistas em voz e fonoaudiólogas clínicas. A avaliadora 1, com 30 anos de experiência, realizou a escuta clínica contextualizada e foi a terapeuta dos pacientes. Dessa forma, conhecia o diagnóstico laringológico, o histórico da disfonia, e realizou a reabilitação vocal de todos os pacientes. A avaliadora 2, com 15 anos de experiência em análise 
auditiva, realizou a escuta cega e não teve acesso a nenhuma informação sobre os pacientes. Ambas as avaliadoras tinham ampla experiência na análise de vozes disfônicas e no uso da escala analógica visual.

\section{Protocolo de avaliação}

Foi realizada a análise perceptivo-auditiva do grau geral de desvio vocal (G), com a utilização da Escala Analógica Visual (EAV) de $100 \mathrm{~mm}$, de uso habitual pelas avaliadoras. Como cada milímetro corresponde a um grau de desvio vocal, a escala oferece 100 possibilidades de graduação. De acordo com estudos prévios $^{(19)}$, existem três valores de corte que determinam quatro faixas de distribuição na EAV: $35,5 \mathrm{~mm}$ (sensibilidade 0,702 e especificidade 1,000); $50,5 \mathrm{~mm}$ (sensibilidade 0,769 e especificidade 1,000) e 90,5 mm (sensibilidade 0,962 e especificidade 0,953 ). Dessa forma, escores de 0 a $35,5 \mathrm{~mm}$ correspondem a variabilidade normal da qualidade vocal; de $35,6 \mathrm{~mm}$ a $50,5 \mathrm{~mm}$, a desvios de leve a moderado; de 50,6 $\mathrm{mm}$ a 90,5 $\mathrm{mm}$, a desvios moderados e de $90,6 \mathrm{~mm}$, a 100 a desvios intensos.

\section{Análise estatística}

Os dados foram tabulados e analisados da seguinte forma: comparação das avaliações de ambas fonoaudiólogas nos momentos pré e pós fonoterapia, tanto para vogal sustentada como para fala encadeada; grau de concordância entre as avaliadoras; diferenças entre as avaliações, de acordo com o gênero, idade, diagnóstico clínico dos pacientes e demanda vocal. Para a análise estatística, adotou-se o nível de significância de 5\% $(0,05)$. Foram utilizados os testes não paramétricos de Wilcoxon e de Igualdade de duas proporções.

\section{RESULTADOS}

Para a tarefa de vogal sustentada, os resultados mostraram que o grau de concordância interavaliadoras foi baixo, tanto no momento pré como no pós fonoterapia. Já para a tarefa de fala encadeada, o grau de concordância foi baixo apenas no momento pós fonoterapia. As avaliadoras apresentaram confiabilidade interna em ambas as tarefas.

A avaliadora 2, que realizou a escuta cega, considerou os desvios maiores em ambos os momentos, durante a tarefa de vogal sustentada, como nos mostram os dados da média da pontuação entre as duas avaliadoras, identificando que houve diferença entre as avaliações (Tabela 1).

Já para a fala encadeada, encontramos diferença entre as avaliadoras somente no momento pós fonoterapia, em que os desvios maiores novamente foram marcados pela avaliadora 2 (Tabela 2).

As variáveis gênero, diagnóstico médico e demanda vocal não apresentaram diferenças nos resultados, indicando que não interferem na avaliação. Porém, a amostra não foi suficientemente ampla para se obter precisão sobre isso.

\section{DISCUSSÃO}

A análise perceptivo-auditiva é a principal forma de avaliação vocal de pacientes disfônicos e um instrumento muito utilizado na realização de pesquisas científicas. Dessa forma, pode ser empregada com finalidades clínicas e científicas, e, muitas vezes, realizada por fonoaudiólogos clínicos. Embora seja uma avaliação subjetiva, o controle adequado dos fatores de interferência dessa análise, como experiência do ouvinte, grau do desvio vocal, protocolo utilizado, parâmetros vocais,

Tabela 1. Valores da análise perceptivo-auditiva da vogal sustentada/E/ realizada pelas avaliadoras 1 e 2 , nos momentos pré e pós fonoterapia

\begin{tabular}{ccccccccc}
\hline & $\begin{array}{c}\text { Vogal } \\
\text { sustentada }\end{array}$ & Média & Mediana & $\begin{array}{c}\text { Desvio- } \\
\text { padrão }\end{array}$ & Mínimo & Máximo & IC & Valor de $p$ \\
\hline \multirow{2}{*}{ Pré } & Aval 1 & 53,82 & 41,5 & 28,09 & 17 & 100 & 11,74 \\
& Aval 2 & 62,82 & 57 & 24,77 & 32 & 100 & 10,35 \\
\hline \multirow{2}{*}{ Pós } & Aval 1 & 22,82 & 21,5 & 11,33 & 7 & 47 & 4,73 \\
& Aval 2 & 51,95 & 48 & 14,36 & 38 & 92 & $6001^{*}$ \\
\hline
\end{tabular}

${ }^{*}$ Valores significativos $(p<0,05)$ - Teste de Wilcoxon

Legenda: Aval = avaliação; IC = Intervalo de Confiança para Média

Tabela 2. Valores da análise perceptivo-auditiva da fala encadeada realizada pelas avaliadoras 1 e 2, nos momentos pré e pós fonoterapia

\begin{tabular}{ccccccccc}
\hline & $\begin{array}{c}\text { Fala } \\
\text { encadeada }\end{array}$ & Média & Mediana & $\begin{array}{c}\text { Desvio- } \\
\text { padrão }\end{array}$ & Mínimo & Máximo & IC & Valor de $p$ \\
\hline \multirow{2}{*}{ Pré } & Aval 1 & 60,32 & 58 & 25,16 & 26 & 100 & 10,51 \\
& Aval 2 & 57,77 & 49,5 & 25,71 & 25 & 100 & 10,74 \\
\multirow{2}{*}{ Pós } & Aval 1 & 18,41 & 14 & 14,04 & 5 & 55 & 5,87 & $<, 334$ \\
& Aval 2 & 43,55 & 15,01 & 15,01 & 18 & 80 & 6,27 \\
\hline
\end{tabular}

*Valores significativos $(p<0,05)$ - Teste de Wilcoxon

Legenda: Aval = avaliação; IC = Intervalo de Confiança para Média 
tipo de estímulo e treinamento do avaliador, torna essa forma de análise robusta e confiável ${ }^{(3,5)}$ Além desses fatores, ainda não se sabe se a escuta contextualizada, em que o fonoaudiólogo conhece desde a queixa vocal e o diagnóstico laringológico até a evolução clínica do paciente em terapia, é um fator de interferência na análise perceptivo-auditiva.

No presente estudo, os principais fatores de interferência na análise perceptivo-auditiva foram devidamente controlados. Dessa forma, optou-se por realizar a análise do grau geral de desvio vocal $(G)$ da escala japonesa GRBAS ${ }^{(20)}$, por ser um parâmetro robusto e confiável ${ }^{(3,11)}$, com a utilização da escala analógica visual, por apresentar maior confiabilidade interavaliadores ${ }^{(13)}$ e por ser mais sensível a pequenas diferenças de marcação que a escala numérica ${ }^{(2)}$.

Os resultados das avaliações, que mostram diferenças, principalmente no momento pós fonoterapia (Tabelas 1 e 2), indicam que, provavelmente, houve influência dos conhecimentos da avaliadora 1 , já que foi a responsável pela terapia fonoaudiológica dos pacientes, tendo acompanhado toda a sua trajetória clínica, desde a avaliação inicial até a alta fonoaudiológica. Essa hipótese mostra a subjetividade envolvida na análise perceptivo-auditiva ${ }^{(21)}$ e reforça a necessidade de se definir o objetivo dessa forma de avaliação, para que se possa escolher a melhor condição de escuta possível.

A discrepância observada nos resultados do momento pós fonoterapia, em que a avaliadora 2 atribuiu maiores pontuações em relação à avaliadora 1 , tanto para o estímulo de vogal sustentada quanto para fala encadeada (Tabelas 1 e 2), demonstra que o acompanhamento do paciente e de sua terapia afeta diretamente a avaliação, principalmente no momento pós fonoterapia, pelo contexto e valor humano na busca por uma mudança de comportamento vocal. Durante o processo de reabilitação vocal, a terapia envolve a modificação de ajustes musculares, revisão de comportamentos vocais mais saudáveis e substituição de hábitos inadequados, além de um processo de adaptação da imagem vocal. Acompanhar todas essas variáveis, seguramente produz um impacto na percepção do clínico, por mais que ele mantenha o saudável distanciamento terapeuta-paciente.

Os avaliadores podem desenvolver padrões de referência internos, de acordo com os modelos de escuta ${ }^{(22,23)}$ Dessa forma, diferenças interavaliadores, principalmente entre profissionais com distintos níveis de experiência, podem ser esperadas. Entretanto, neste estudo, ambas as fonoaudiólogas tinham experiência clínica e científica na avaliação de vozes disfônicas e amplo treinamento auditivo.

O estudo que identificou os valores de corte para a escala analógica visual no $\mathrm{Brasil}^{(19)}$, obteve alto índice de sensibilidade e especificidade, utilizando o grau geral do desvio vocal (G) para sua análise, demonstrando ser um bom instrumento para avaliação perceptiva do desvio geral da voz. Essa escala é composta por três valores de corte e quatro faixas de distribuição ${ }^{(15,19)}$. Esses achados diferem de outros protocolos que também utilizam a escala analógica visual em sua concepção, como é o caso do CAPE-V, que possui diferentes faixas de distribuição ${ }^{(14)}$.

A utilização de diferentes tarefas vocais para realização da avaliação perceptivo-auditiva é um viés que vem sendo explorado nos últimos anos e pesquisas mostram que os escores na marcação do grau geral do desvio vocal têm sido maiores para as tarefas de vogais sustentadas, em comparação com as de fala encadeada ${ }^{(10,12)}$. Nessas pesquisas, foram observados maiores desvios na avaliação das vogais sustentadas em relação à fala encadeada, indicando que os dois tipos de tarefas devem ser utilizados para o julgamento clínico na avaliação perceptivo-auditiva da intensidade da disfonia ${ }^{(24)}$, concordando com os achados desse estudo.

Na produção da fala encadeada, a emissão é muito próxima da fala natural dos pacientes, pois há maior interação entre fonte sonora e filtro (articulação, velocidade de fala, ritmo), enquanto que na vogal sustentada há apenas informações importantes sobre a fonte sonora. De alguma forma, as informações contidas na fala encadeada fizeram com que o julgamento entre as duas avaliadoras fosse mais próximo na análise pré fonoterapia. É difícil levantar hipóteses sobre as diferenças observadas na avaliação da vogal sustentada e, talvez, nesse segmento sonoro, as preferências pessoais e o sistema interno de referências tenha tido maior peso no julgamento perceptivo.

Pudemos observar, pelos dados, que somente no momento pós fonoterapia houve maior distanciamento entre as análises, o que pode ser consequência direta da escuta contextualizada. Interessante ressaltar que a análise das avaliadoras no momento pré fonoterapia foi muito próxima, mesmo numa escala com 100 possibilidades de marcação. Isso mostra que a experiência clínica das avaliadoras na análise de pacientes disfônicos favorece fortemente a confiabilidade interavaliadores.

Observando mais atentamente os resultados e relacionando-os com o grau de alteração encontrado na escala analógica visual $^{(18)}$, verificamos, mais uma vez, a interferência do tipo de tarefa durante a análise perceptivo-auditiva. Pode-se inferir que a avaliadora 1 , conhecendo os dados e o momento de terapia dos pacientes, identificou mudanças vocais, enquanto a avaliadora 2, realizando a escuta cega, não notou diferença entre ambos os momentos, para vogal sustentada (Tabela 1), porém observou uma leve mudança nas vozes na tarefa de fala encadeada (Tabela 2).

Discrepâncias entre os resultados de avaliações vocais clínicas e avaliações realizadas em ambientes de pesquisa já foram relatadas, indicando que alguns vieses clínicos, como local de escuta ou conhecimento clínico, podem ter afetado os resultados ${ }^{(18)}$. De acordo com o estudo, em um ambiente clínico os fonoaudiólogos têm acesso à história médica do paciente no decorrer da anamnese, antes da realização da avaliação vocal. Já em um ambiente de pesquisa, a avaliação perceptivo-auditiva geralmente é realizada sem esse contexto, a informação clínica pode ser retida e as amostras de voz randomizadas, para posterior avaliação ${ }^{(18)}$. Podemos, nesse âmbito, inferir 
que o conhecimento do percurso clínico altera os resultados das avaliações e pode aumentar ou diminuir a intensidade do desvio vocal.

Quando comparou-se a análise perceptivo-auditiva sem o conhecimento do diagnóstico laringológico e após ser revelado aos avaliadores, verificou-se um aumento na marcação da intensidade do desvio vocal, quando em posse do diagnóstico ${ }^{(17)}$. Por isso, recomenda-se que as avaliações sejam feitas em situações consistentes, tanto pré como pós fonoterapia, para garantir sua validade. O conhecimento do contexto clínico, já incluindo diagnóstico e também outros dados, como gênero, idade e informações do percurso terapêutico dos pacientes, deve ser mais um viés a ser evitado na avaliação perceptivo-auditiva.

Estímulos apresentados pela primeira vez, sem nenhum contexto sobre o paciente e depois de várias semanas, com informações complementares, em particular, se a voz era pré ou pós fonoterapia, mostraram que o julgamento da análise perceptivo-auditiva foi mais dependente da informação contextual (pré/pós tratamento) do que apenas da escuta do sinal sonoro e que somente testes cegos podem fornecer resultados confiáveis em uma análise vocal ${ }^{(25)}$, evidenciando, assim como ora é realizado, a importância de se delinear a forma de avaliação, para se evitar mais um viés.

O desenho do experimento é crucial para que se saiba exatamente o que se está avaliando. Seguramente, o terapeuta do paciente não é um avaliador imparcial. Pesquisas científicas que envolvam a análise auditiva precisam ter um desenho de experimento bastante claro e definido. Assim, se a pesquisa tiver o objetivo de avaliar a amostra vocal do ponto de vista clínico, os avaliadores devem ter acesso às informações clínicas dos pacientes. Por outro lado, se o objetivo da pesquisa é o de analisar exclusivamente o grau de desvio do sinal sonoro, nenhuma informação precisa ser oferecida aos avaliadores e o terapeuta que fez o acompanhamento dos pacientes, cujas vozes façam parte da amostra, deve ser excluído da avaliação. A necessidade de uniformizar procedimentos para a avaliação clínica de voz é necessária ${ }^{(18,25)}$, na tentativa de elucidar potenciais vícios na avaliação perceptivo-auditiva vocal.

Em suma, os resultados da pesquisa mostraram que o contexto clínico influencia significativamente a avaliação da intensidade do desvio vocal, em diferentes tarefas vocais, principalmente no momento pós fonoterapia. Porém, temos como limitações deste estudo, o baixo número de amostras vocais que compuseram a pesquisa e o baixo número de avaliadores. Em estudos futuros, sugerimos a ampliação da amostra vocal e a utilização de mais avaliadores para ambas as análises, ou seja, escuta contextualizada e escuta cega.

\section{CONCLUSÃO}

A situação de escuta que envolve o conhecimento ou não do contexto clínico tem impacto direto na percepção de uma disfonia, mesmo em fonoaudiólogos experientes. A vogal sustentada sofre maior variabilidade interavaliadores do que a fala encadeada.

\section{REFERÊNCIAS}

1. Kreiman J, Gerratt BR, Kempster GB, Erma A, Berke GS. Perceptual evaluation of voice quality: review, tutorial, and a framework for future research. J Speech Hear Res. 1993;36(1):21-40.

2. Wuyts FL, De Bodt MS, Heyning PHV. Is the reliability of a visual analog scale higher than an ordinal scale? An experiment with the GRBAS scale for the perceptual evaluation of dysphonia. J Voice. 1999;13(4):508-17.

3. Oates J. Auditory-perceptual evaluation of disordered voice quality. Folia Phoniatr Logop. 2009;61(1):49-56.

4. Carding PN, Awilson J, Mackenzie K, Deary IJ. Measuring voice outcomes: state of the science review. J Laringol Otol. 2009;123(8):823-9.

5. Patel S, Shrivastav R. Perception of dysphonic vocal quality: some thoughts and research update. ASHA SID-3 Newsletter - Voice Voice Disorders. 2007;17(2):3-6.

6. Behlau M, Madazio G, Feijó D, Pontes P. Avaliação de voz. In: Behlau M. Voz: o livro do especialista. Vol. 1, Avaliação de voz. Rio de Janeiro: Revinter; 2001. p. 85-246.

7. Speyer R, Wieneke GH, Dejonckere PH. Documentation of progress in voice therapy: perceptual, acoustic, and laryngostroboscopic findings pretherapy and posttherapy. J Voice. 2004;18(3):325-40.

8. Bele IV. Reliability in perceptual analysis of voice quality. J Voice. 2005;19(4):555-73.

9. Eadie TL, Doyle, PC. Direct magnitude estimation and interval scaling of pleasantness and severity in dysphonic and normal speakers. J Acoust Soc Am. 2002;112(6):3014-21.

10. Zraick RI, Wendel K, Smith-Olinde L. The effect of speaking task on perceptual judgment of the severity of dysphonic voice. J Voice. 2005;19(4):574-81.

11. Eadie TL, Baylor CR. The effect of perceptual training on inexperienced listeners' judgments of dysphonic voice. J Voice. 2006;20(4):527-44.

12. Wolfe V, Cornell R, Fitch J. Sentence/vowel correlation in the evaluation of dysphonia. J Voice. 1995;9(3):297-303.

13. Kreiman J, Gerratt BR, Ito M. When and why listeners disagree in voice quality assessment tasks. J Acoust Soc Am. 2007;122(4):2354-64. 14. Behlau M. Consensus Auditory- Perceptual Evaluation of Voice (CAPE-V), ASHA 2003. Refletindo sobre o novo. Rev Soc Bras Fonoaudiol. 2004;9(3):187-9.

15. Simberg S, Sala E, Laine A, Rönnemaa AM. A fast and easy screening method for voice disorders among teacher students. Logoped Phoniatr Vocol. 2001;26(1):10-16.

16. Yamasaki R, Leão SHS, Madazio G, Padovani M, Azevedo R. Análise perceptivo-auditiva de vozes normais e alteradas: escala analógica visual. In: Anais do 15. Congresso Brasileiro de Fonoaudiologia; 7. Congresso Internacional de Fonoaudiologia; 16-20 out 2007; Gramado, Brasil. São Paulo: Sociedade Brasileira de Fonoaudiologia; 2007. p. 16-20.

17. Eadie T, Sroka A, Wright DR, Merati A. Does knowledge of medical 
diagnosis bias auditory-perceptual judgments of dysphonia? J Voice. 2011;25(4):420-9.

18. Solomon NP, Helou LB, Stojadinovic A. Clinical versus laboratory ratings of voice using the CAPE-V. J Voice. 2011;25(1):e7-14.

19. Yamasaki R, Leão SHS, Madazio G, Padovani M, Azevedo R, Behlau M. Correspondência entre Escala Analógico-Visual e a Escala Numérica na avaliação perceptivo-auditiva de vozes. In: Anais do 16. Congresso Brasileiro de Fonoaudiologia; 24-27 set 2008; Campos de Jordão, Brasil. São Paulo: Sociedade Brasileira de Fonoaudiologia; 2008. p. 24-7.

20. Hirano M. Clinical examination of voice. New York: Springer; 1981. (Disorders of human communication, 5),

21. Chan KM, Yiu EM. The effect of anchors and training on the reliability of perceptual voice evaluation. J Speech Lang Hear Res. 2002;45(1):111-26.
22. Eadie TL, Kapsner M, Rosenzweig J, Waugh P, Hillel A, Merati A. The role of experience on judgments of dysphonia. J Voice. 2010;24(5):564-73.

23. Hakkesteegt MM, Brocaar MP, Wieringa MH, Feenstra L. The relationship between perceptual evaluation and objective multiparametric evaluation of dysphonia severity. J Voice. 2008;22(2):138-45.

24. Maryn Y, Roy N. Sustained vowels and continuous speech in the auditory-perceptual evaluation of dysphonia severity. J Soc Bras Fonoaudiol. 2012;24(2):107-12.

25. Ghio A, Révis J, Merienne S, Giovanni A. Top-down mechanisms in dysphonia perception: the need for blind tests. J Voice. 2013;27(4):481-5. 